Journal of Computer Science 6 (3): 269-278, 2010

ISSN 1549-3636

(C) 2010 Science Publications

\title{
Improving Triple Play Services Using Multi Protocol Label Switching Technology
}

\author{
Waheed Yasin and Hamidah Ibrahim \\ Department of Communication Technology and Network, \\ Faculty of Computer Science and Information Technology, \\ University Putra Malaysia, Serdang, Selangor Darul Ehsan, Malaysia
}

\begin{abstract}
Problem statement: Traditional IP networks have many limitations such as routing tables, which can be complex and time consuming. These limitations affect the performance of the network in some applications of triple play services (i.e., voice, video and data) which are characterized as time sensitive applications. Thus, Multi Protocol Label Switching (MPLS) technology has been proposed to speed up the traffic flow in the network using labels. Approach: In this study, an experiment using the Network Simulator NS-2 was performed to evaluate the impact of MPLS technology on the Triple Play Services based on the average throughput of the network, total number of packets received at destination nodes and packet loss rates and this is compared to that provided by traditional IP networks. Results: The results showed that MPLS performs better since it utilizes all the available paths to the destinations. Conclusion: MPLS allows Internet Services Providers (ISPs) to provide better triple play services for end-users.
\end{abstract}

Key words: IP Networks, VoIP, IPTV, FTP, NS-2

\section{INTRODUCTION}

Nowadays, the use of the Internet becomes more complex. Everyday this network becomes bigger and bigger and also the services, which are offered on the Internet, are more challenging. Triple Play Services is the term used to describe the combination of voice, video and data transmission services. These challenges impress Internet Service Providers (ISPs) to keep upgrading their network infrastructures to meet the requirements of these services.

Traditional Internet Protocol (IP) networks (Maufer, 1999) offer little predictability of service, which is unacceptable for applications such as telephony, as well as for emerging and future real-time applications. Thus, in order to offer these services, there must be a way for guaranteeing the Quality of Service (QoS). Routing table of IP router can be complex and time consuming. Thus, the performance of network with some of triple play application in heavy traffic environments will be affected (Porwal et al., 2008).

Multi Protocol Label Switching (MPLS) technology (Martin, 2008) offers the Quality of Service (QoS) that guarantees data communication service as Frame Relay (FR) and Asynchronous Transfer Mode
(ATM) do; however without requiring the use of any dedicated lines. That is due to its ability to speed up the traffic flow by using labels. These advantages make MPLS plays a key role in Next Generation Networks (NGN), which aims to provide one network for multiple services rather than one network for one service and different networks for different services.

The aims of this study are to evaluate the impact of MPLS technology on Triple Play Services based on the average throughput of the network, total number of packets received at destination nodes and packet loss rates; and to compare its performance to that provided by the IP networks.

IP networks: Conventional IP networks use routing, which is the process of selecting paths in a network along which to send network traffic. Each router in the network has to make independent routing decisions for each incoming packet. When a packet arrives at a router, the router has to consult its routing table to find the next hop for that packet based on the packet destination address in the packets IP header. To build routing tables each router runs IP routing protocols like Border Gateway Protocol which has been identified by the Internet Engineering Task Force

Corresponding Author: Waheed Yasin, Department of Communication Technology and Network,

Faculty of Computer Science and Information Technology, University Putra Malaysia, 43400 UPM Serdang,Selangor Darul Ehsan, Malaysia 
(IETF) Request For Comment (RFC4271) (Goralski, 2009), Open Shortest Path First (OSPF) (RFC2328) (Tiwari and Sahoo, 2007) or Intermediate System-toIntermediate System (IS-IS) (RFC3784) (Sridharan et al., 2005). When a packet traverses through the network, each router performs the same steps of finding the next hop for the packet. For more details regarding IP networks and routing protocols, readers may refer to (Doyle and Carroll, 2001).

IP networks use Internet Protocol Address (IP Address), which is a 32 bit unique number assigned to each computer's or other device's network interface(s) which are active on a network supporting IP, in order to distinguish each network interface from every other network interface anywhere on the network. For more details regarding IP address, readers may refer to (Comer, 2000).

Traditional IP network has many weaknesses which make it unable to meet new applications' requirements. One of these weaknesses is that it does not consider capacity constraints and traffic characteristics when routing decisions are made, which results in some segments of a network become congested while other segments along alternative routes become underutilized and worse than that is that traditional routing protocols will continue forwarding traffic across these paths until packets are dropped. Figure 1 illustrates the traffic flow in OSPF network. It can be noticed that the shortest path is congested while others paths are underutilized.

Besides, traditional IP network has limited capability to deal with addressing information because it depends only on the destination IP address carried on the packet header. Because all traffic to the same IP destination header is usually treated similarly, several difficulties appear. For example, it becomes difficult to perform traffic engineering on IP networks. Also, it is more difficult to manage flows of data in a packet switched network than in a circuit switched network because each packet is manipulated individually. Moreover, routing table look up in an IP router can be complex and time consuming, which reduces the performance of IP network and this clearly appears in heavy traffic environments such as triple play services.

MPLS technology: MPLS is a standard approved technology for speeding up network traffic flow and making it easier to manage. MPLS can accommodate highly interactive application flows with low delay and packet loss threshold. MPLS involves setting up a specific path for a given sequence of packets called Label Switched Path (LSP), identified by a label added to each packet, thus saving the time needed for a router to look up the address to the next node to forward the packet to.

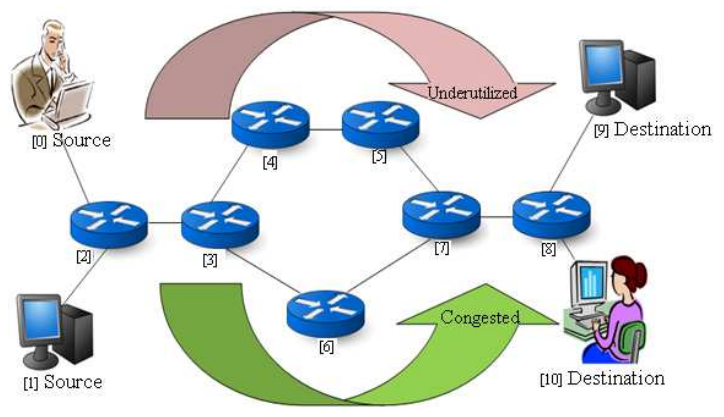

Fig. 1: Traffic flows in an OSPF network

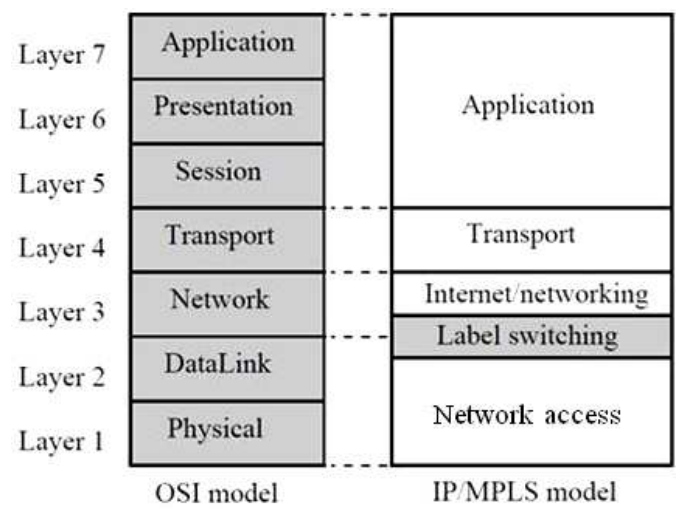

Fig. 2: MPLS location according to the OSI model

MPLS is called multiprotocol because it works with the IP, ATM (Cuthbert and Sapanel, 1993) and FR (Buckwalter, 2000) network protocols. Also, the extension Generalized MPLS (GMPLS) (RFC3471) (Banerjee et al., 2001). Also, it is known as Multiprotocol Lambda Switching (MLS) and has been proposed for optical networks. MPLS operates at the Open System Interconnection (OSI) reference model layer which is generally considered to lie between Layer 2 (data link layer) and Layer 3 (network layer) and thus is sometimes defined as a "Layer 2.5" protocol. Thus, MPLS is a set of procedures for combining the performance, QoS and traffic management of the Layer 2 label-swapping model with the scalability and flexibility of Layer 3 routing functionality. Figure 2 illustrates where the MPLS is located according to the OSI model.

MPLS domain: The MPLS domain can be divided into MPLS core and MPLS edge. The core consists of nodes neighboring only to MPLS capable nodes, while the edge consists of nodes neighboring both MPLS capable and incapable nodes. The nodes in the core of the MPLS domain are called Label Switch Routers (LSRs), however the nodes in the MPLS edge are called Label Edge Routers (LERs). 


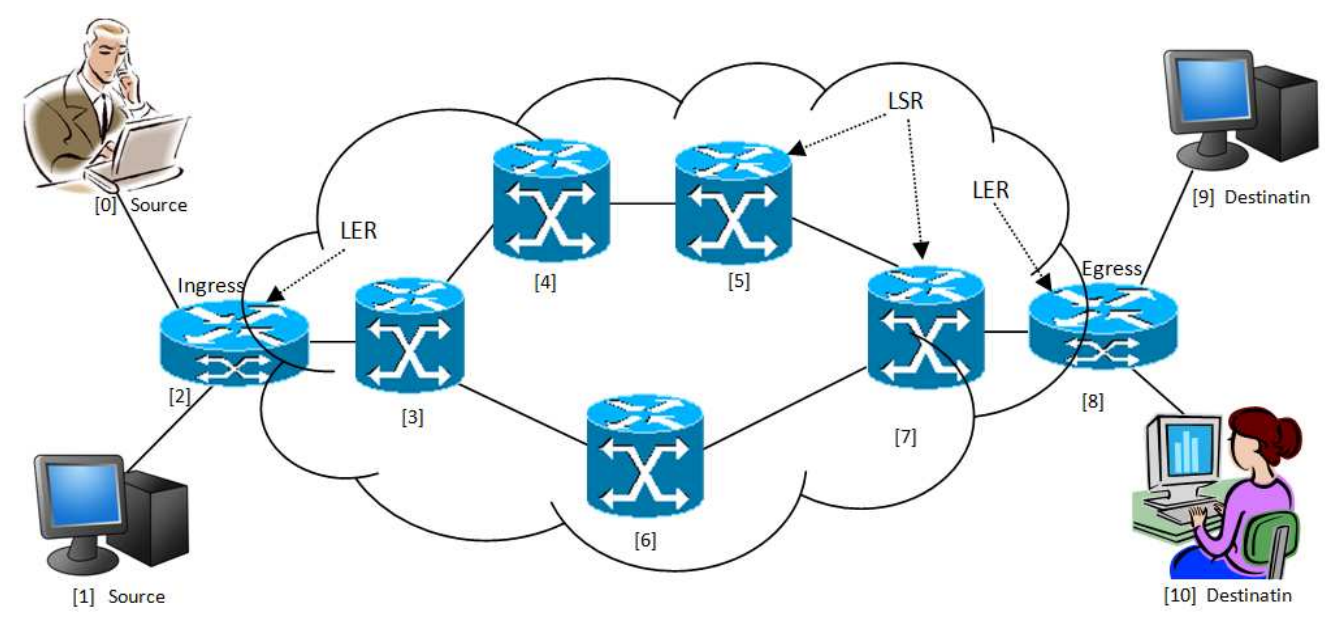

Fig. 3: MPLS Domain

The LER is called an MPLS Ingress node if its role is handling traffic as it enters the MPLS domain; however, it is called an MPLS Egress node if its role is handling traffic as it leaves the MPLS domain. Figure 3 illustrates the MPLS domain.

A main concept in MPLS is the separation of an IP router's function into two parts: forwarding and control (El Hachimi et al., 2004). The forwarding part is responsible for how data packets are relayed between IP routers, using label swapping. The control part consists of network layer routing protocols to broadcast routing information between routers and label binding procedures for converting this routing information into the forwarding tables needed for label switching. This separation enables each component to be developed and modified independently. An important point of MPLS that should be noted is that MPLS is not a routing protocol; however, it is a fast forwarding mechanism which is designed to work with existing IP routing protocols such as OSPF or BGP.

MPLS label: MPLS label (RFC3032) is a 32 bit which consists of the following fields, which are illustrated in Fig. 4 and listed as follows:

- A label field which is 20 bits and carries the actual value of the MPLS label

- Class of service field or sometimes called experimental field, which consists of 3 bits can influence the queuing and remove algorithms, which are applied to the packet when it is transmitted through the MPLS network

- A single bit field shows a hierarchical label stack

- $\quad$ Time To Live (TTL) field which is 8 bits that gives the usual IP time to live functionality

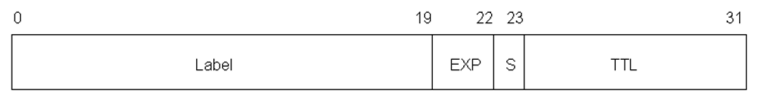

20-bit Label Value

3-bit Experimental Field

-bit bottom-of-stack indicator

Fig. 4: MPLS label

\begin{tabular}{|l|l|l|l|l|}
\hline Layer 2 Header & Top Label & $\ldots$ & Bottom Label & Layer 3 Header \\
\hline
\end{tabular}

Fig. 5: Label location

The label is located between the data link layer (Layer 2) header and network layer (Layer 3) header. The top of the label stack appears first in the packet and the bottom appears last. The network layer packet immediately follows the last label in the label stack. Label location is illustrated in Fig. 5.

LDP: The Label Distribution Protocol (LDP) (RFC3036) has been defined for the purpose of distributing labels in an MPLS environment. LDP is a set of procedures and messages by which LSRs establish Label Switched Paths (LSPs) through a network by mapping network layer routing information directly to data link layer switched paths, as shown in Fig. 6. Allocation of label in MPLS network is done by the downstream peers, where downstream is explained with respect to routing. There are two types of label allocation: Downstream on demand and unsolicited downstream. In downstream on demand label distribution mode, MPLS architecture allows an LSR to explicitly request from its next hop a particular Forward Equivalent Class (FEC) (i.e., a set of packets that are treated identically by an LSR) a label binding for that FEC, where the upstream LSR is responsible for requesting a label binding. 


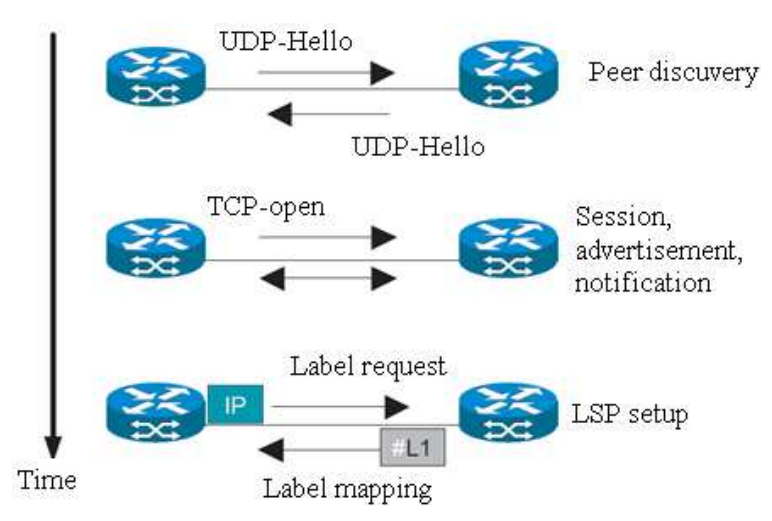

Fig. 6: Label distribution protocol

Downstream on demand label distribution mode is useful in ATM networks where combining of LSPs is not possible. In unsolicited downstream, the MPLS architecture allows an LSR to distribute label bindings to LSRs that have not explicitly requested them. Thus, the downstream LSR is responsible for advertising a label mapping to upstream LSRs (Feher et al., 2002).

LSP: A Label Switched Path (LSP) is a path through an MPLS network, which is set up by a signaling protocol such as LDP, Resource Reservation Protocol (RSVP) (RFC2205) (Chow and Leon-Garcia, 1999) and its extension for traffic engineering (RSVP-TE) (RFC3209) (Lee et al., 2007), BGP or Constraint-based Routing Label Distribution Protocol (CRLDP) (RFC3212) (Szviatovszki et al., 2002). The path is set up based on criteria in the FEC. LSP is an ingress-toegress switched path built by MPLS nodes. A key point of LSP is that LSP is unidirectional. Thus, it enables a packet to be label switched through the MPLS network from one endpoint to another. Since bidirectional communication is typically desired, the signaling protocols can set up an LSP in the other direction as an atonement for this. There are two types of label distribution control modes in the MPLS architecture in order to establish an LSP, which are independent label distribution mode and ordered label distribution mode (Wang and Li, 2008). In the former, each LSR makes an independent decision for binding a label to a particular FEC and distribute that biding to its neighbors; while, in the later, LSR only binds in response to a label binding request.

\section{MATERIALS AND METHODS}

This research is a quantitative research, where we run an experiment to evaluate the impact of MPLS technology on triple play services.

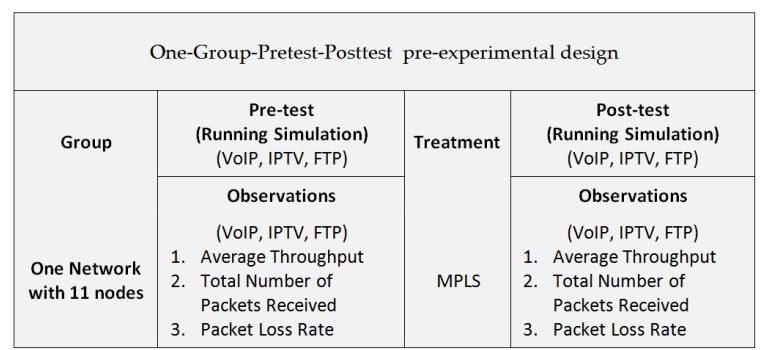

Fig. 7: Research experimental design

Research methodology design: The experimental design for this study is called "One-Group PretestPosttest Design", which is a pre-experimental design. We used one network with 11 nodes in our simulation, which is considered as one group. The number of nodes has been chosen randomly as in (Porwal et al., 2008). In the pre-test stage, three subtests have been applied on the network, which have the traditional IP characteristics. Each subtest was applied with different applications, which are IPTV, VoIP and FTP. In the post-test stage, the same subtests with the same applications have been applied to the network but after implementing the treatment, which is in our case the MPLS technology. These tests have been used to show the effect of MPLS on the triple play services. The average throughput of the network, the total number of packets received at the destination nodes and packet loss rates have been observed to evaluate the impact of the MPLS technology. The research design is summarized in Fig. 7.

Simulation tool: The simulation tool which has been used in this research was based on Network Simulator (NS) version 2.33. NS-2 is an open source software, which is available for public and can be obtained from the Information Sciences Institute (ISI) web site http://www.isi.edu/nsnam/ns/. A key point has to be mentioned is that a patch file which is specially created to simulate the reservation control protocol for traffic engineering RSVP-TE in MPLS has been used. The patch file is called "MNS-RSVP" and the most important thing which should be considered about this patch file is that it is only exclusive for NS-2 version 2.33. Thus, it might not work with other versions of NS-2. Readers may refer to (Issariyakul and Hossain, 2008) for more information about NS-2. A free documentations for NS-2 are available online such as on the ISI web site, also it might be downloaded using this URL:http://www.isi. edu/nsnam/ns/doc/ns_doc.pdf. 


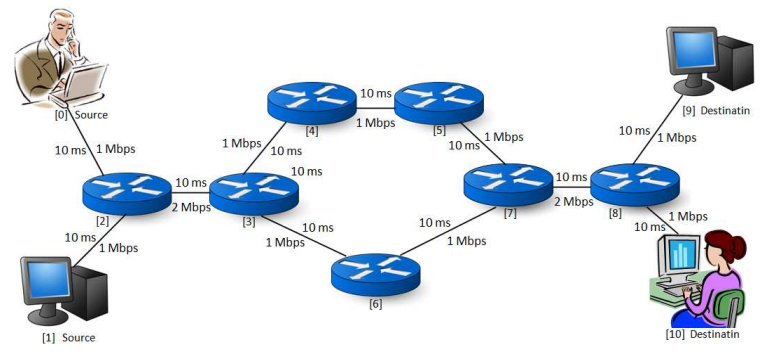

Fig. 8: Network topology

Table 1: NS-2 simulation settings for IP network

\begin{tabular}{ll}
\hline Item & Setting \\
\hline All Nodes & $\begin{array}{l}\text { IP capable } \\
\text { duplex }\end{array}$ \\
All links & \\
Bandwidth of the following links: & $1 \mathrm{Mbps}$ \\
$0-2,1-2,3-4,3-6,4-5,5-7,6-7,8-9$, and $8-10$ & $2 \mathrm{Mbps}$ \\
Bandwidth of 2-3 and 7-8 links & $10 \mathrm{~ms}$ \\
Link Propagation Delay & DropTail \\
Queuing Type &
\end{tabular}

Table 2: NS-2 Simulation settings for MPLS network

\begin{tabular}{ll}
\hline Item & Setting \\
\hline Node 0, 1,9 and 10 & IP capable \\
Node 2-8 & MPLS capable \\
All links & Duplex-rsvp \\
Bandwidth of the following links: 0-2, 1-2, 3-4, & 1 Mbps \\
3-6, 4-5, 5-7, 6-7, 8-9, and 8-10 & \\
Bandwidth of 2-3 and 7-8 links & $2 \mathrm{MB}$ \\
Link propagation delay & $10 \mathrm{~ms}$ \\
Queuing type & DropTail \\
\hline
\end{tabular}

Network topology: Figure 8 illustrates the network topology which has been used in this work. In pre-test stage, all links were setup as duplex with $10 \mathrm{~ms}$ propagation delay and using DropTail queuing system, which serve packets on a First Come First Serve (FCFS) basis. In post-test stage, all links were configured as duplex-rsvp with the same propagation delay as in the pretest stage which is $10 \mathrm{~ms}$. The bandwidths of the links were set to $1 \mathrm{Mbps}$, except the bandwidths of the links 2-3 and 7-8, which they were set to 2 Mbps. NS-2 simulation settings for the traditional IP are summarized in Table 1. Table 2 summarizes NS-2 simulation settings for MPLS. A key point should be noted here is that all the simulation settings were chosen randomly.

Running the simulations: In order to evaluate the performance of MPLS technology, the simulations have been run three times for different applications, which are VoIP, IPTV and FTP.

VoIP scenario: Voice over Internet Protocol (VoIP) (Karapantazis and Pavlidou, 2009) is a general term for describing transmission technologies for voice delivery over IP networks such as the Internet. Thus, VoIP sometimes is called Internet telephony (Mortada and Probst, 2001). For more clarification, Internet telephony refers to voice communication services that are transported via the Internet, rather than the Public Switched Telephone Network (PSTN). This can be performed by a procedure including basic steps involved in originating an Internet telephone call which are analog voice signal conversion to digital format and breaking up of the signal into IP packets for transmission over the Internet and this process is reversed at the receiver. In this research, we have simulated the VoIP traffic using the Pareto On/Off Traffic Generator (POO Traffic) which is a traffic generator (an application) embodied in the Objectoriented Tool command language class (OTcl) Application/Traffic/Pareto of NS-2. Packets are sent at a fixed rate during on periods and no packets are sent during off periods. Both on and off periods are taken from a Pareto distribution with constant size packets. These sources can be used to generate aggregate traffic that exhibits long range dependency. In this research, two POO traffic generators have been used on two User Datagram Protocol (UDP) connections. The first POO generator's source was attached to Node 0 and its destination was Node 10; while the second was attached to Node 1 and its destination was Node 9.

IPTV scenario: Internet Protocol Television (IPTV) is a technology for delivering digital television service over IP networks such as the Internet instead of being delivered through traditional radio frequency broadcast, satellite signal and Cable Television (CATV) formats (Simpson, 2008). IPTV services can be classified into three main groups: live television, timeshifted programming and Video on Demand (VoD). In this research, we have simulated the IPTV traffic using the Constant Bit Rate (CBR), which is a term used in telecommunications relating to the QoS. Two CBR traffic generators have been used on two UDP connections. The first generator's source was attached to Node 0 and its destination was Node 10; while the second was attached to Node 1 and its destination was Node 9.

FTP scenario: File Transfer Protocol (FTP) (RFC959) (Loshin, 2003) is a standard network protocol used to exchange files over a TCP/IP based network, such as the Internet. FTP works in the same way as Hypertext Transfer Protocol (HTTP) for transferring web pages from a server to a user's browser and Simple Mail Transfer Protocol (SMTP) for transferring electronic mail over Internet. FTP is built on a client-server 
architecture. In this research, two FTP traffic generators have been used on two TCP connections. The first FTP traffic generator source was attached to Node 0 and its destination was Node 10; however the second was attached to Node 1 and its destination was Node 9.

\section{RESULTS}

The results which have been gathered from running the simulations for 5 seconds are presented according to the pre-test and post-test for the different three scenarios (VoIP, IPTV and FTP) for both traditional IP and MPLS networks. A snapshot of traffic flows in IP network is illustrated in Fig. 9; while Fig. 10 illustrates a snapshot of traffic flows in MPLS network.

The throughput is measured by the number of bits transferred through a system per time unit. The average throughput is calculated by Eq. 1 :

Average throughput $=\frac{\sum_{i}^{\text {No.of Flows }} \text { Flow }(i)_{\text {throughput }}}{\text { No.of flows }}$

where, Flow(i) $)_{\text {throughput }}$ is the throughput of flow number $\mathrm{i}$; in our case number of flows is 2 .

The packet loss rate is calculated as the total number of packets have been dropped to the total number of packets have been sent.

IPTV scenario: Figure 11 illustrates the performance of MPLS and IP networks in IPTV scenario.

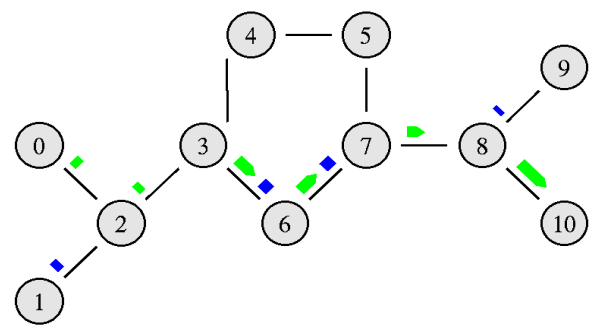

Fig. 9: A snapshot of traffic flows in IP network in NS2 environment

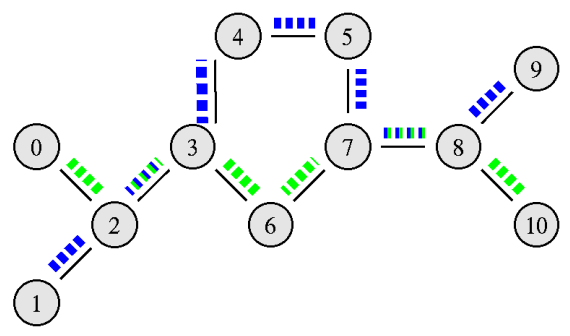

Fig. 10: A snapshot of traffic flows in MPLS network in NS-2 environment
Table 3 summarizes the packet loss rate for both IP network and MPLS network in IPTV scenario; while Table 4 summarizes the total number of IPTV packets received at the destination nodes Node 9 and Node 10 in IP and MPLS networks.

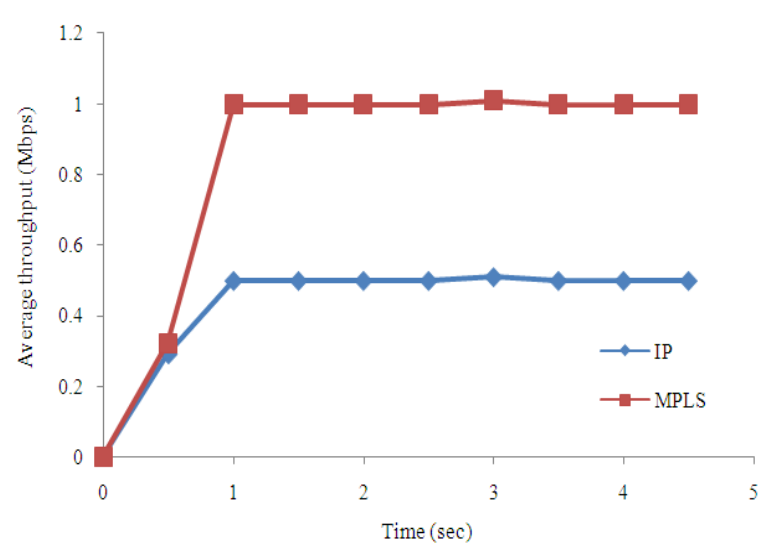

Fig. 11: The performance of IP and MPLS networks in IPTV scenario

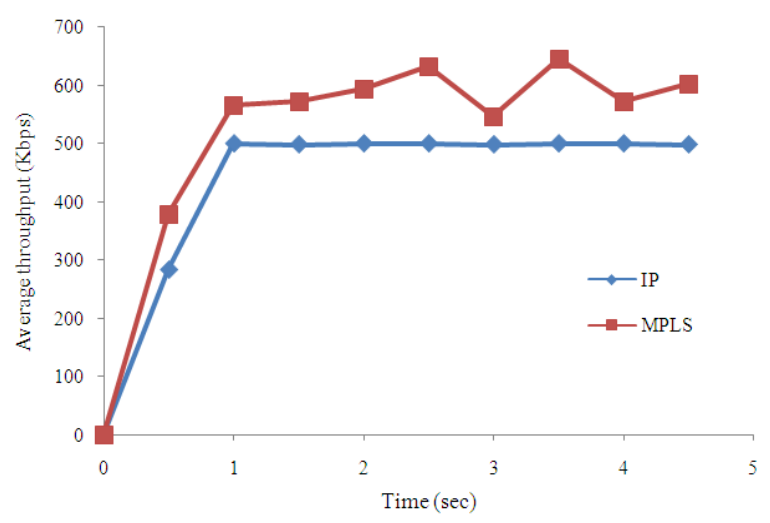

Fig. 12: The performance of IP and MPLS networks in VoIP scenario

Table 3: Packet loss rate for IP and MPLS networks in IPTV scenario

\begin{tabular}{lllll}
\hline $\begin{array}{l}\text { Network } \\
\text { type }\end{array}$ & $\begin{array}{l}\text { Simulation } \\
\text { time }(\mathrm{sec})\end{array}$ & $\begin{array}{l}\text { Total No. } \\
\text { of sent } \\
\text { packets }\end{array}$ & $\begin{array}{l}\text { Total No. } \\
\text { of dropped } \\
\text { packets }\end{array}$ & $\begin{array}{l}\text { Packet } \\
\text { loss rate } \\
(\%)\end{array}$ \\
\hline IP network & 5 & 20835 & 16943 & 81.32 \\
MPLS network & 5 & 22626 & 16619 & 73.45 \\
\hline
\end{tabular}

Table 4: Total number of IPTV packets received at destination nodes

\begin{tabular}{llll}
\hline & $\begin{array}{l}\text { No. of packets received at } \\
\text { destination nodes }\end{array}$ & $\begin{array}{l}\text { Total } \\
\text { No. of } \\
\text { packets } \\
\text { received }\end{array}$ \\
\hline Network type & Node 9 & Node 10 & re----- \\
\hline IP network & 265 & 196 & 461 \\
MPLS network & 454 & 442 & 896 \\
\hline
\end{tabular}


VoIP scenario: Similar to the IPTV scenario, two VoIP traffic generators have been applied. Figure 12 illustrates the average throughput of the two flows in both networks IP and MPLS.

Table 5 summarizes the packet loss rate for both IP network and MPLS network in VoIP scenario; while Table 6 summarizes the total number of VoIP packets received at the destination nodes Node 9 and Node 10 in IP and MPLS networks.

FTP scenario: Similar to the previous scenarios, two FTP traffic generators have been applied. Figure 13 illustrates the performance of MPLS and IP networks in FTP scenario.

Table 7 summarizes the total number of FTP packets received at the destination nodes Node 9 and Node 10 in IP and MPLS networks.

\begin{tabular}{lllll}
\multicolumn{5}{l}{ Table 5: Packet loss rate for IP and MPLS networks in VoIP scenario } \\
\hline & $\begin{array}{l}\text { Simulation } \\
\text { time }(\mathrm{sec})\end{array}$ & $\begin{array}{l}\text { Total No. } \\
\text { of sent } \\
\text { packets }\end{array}$ & $\begin{array}{l}\text { Total No. } \\
\text { of dropped } \\
\text { packets }\end{array}$ & $\begin{array}{l}\text { Packet } \\
\text { loss } \\
\text { rate }(\%)\end{array}$ \\
Network type & 5 & 19451 & 690 & 3.55 \\
IP network & 5 & 22207 & 0 & 0.00 \\
MPLS network & 5 & &
\end{tabular}

Table 6: Total number of VoIP packets received at destination nodes

\begin{tabular}{llll}
\hline & $\begin{array}{l}\text { No. of packets received at } \\
\text { destination nodes }\end{array}$ & $\begin{array}{l}\text { Total No. } \\
\text { of packets } \\
\text { received }\end{array}$ \\
\hline Network type & Node 9 & Node 10 & 2848 \\
\hline IP network & 1513 & 1335 & 3398 \\
\hline MPLS network & 1655 & 1743 & \\
\hline
\end{tabular}

Table 7: Total number of FTP packets received at destination nodes

\begin{tabular}{llll}
\hline & $\begin{array}{l}\text { No. of packets received at } \\
\text { destination nodes }\end{array}$ & $\begin{array}{l}\text { Total No. } \\
\text { of packets } \\
\text { received }\end{array}$ \\
\hline Network type & Node 9 & Node 10 & 534 \\
\hline IP Network & 267 & 267 & 977 \\
\hline MPLS Network & 463 & 514 & \\
\hline
\end{tabular}

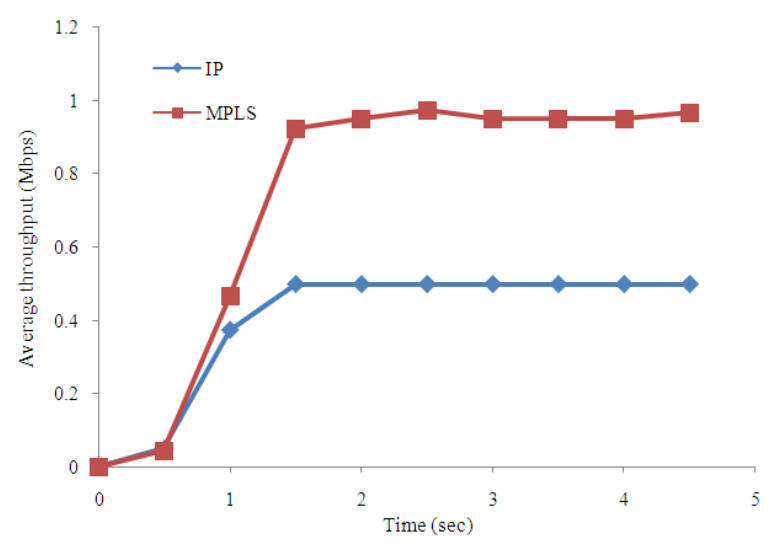

Fig. 13: The performance of IP and MPLS networks in FTP scenario

\section{DISCUSSION}

During running the simulations for all the three scenarios, we noticed that in IP network the traffic went through one path (2-3-6-7-8), which is the shortest path however in MPLS network the traffic went through two paths (2-3-6-7-8) and (2-3-4-5-7-8). By comparing Fig. 9 and 10, it can be observed that MPLS utilizes the paths which are underutilized when the shortest path is congested.

IPTV scenario: Referring to Fig. 11, we observed that MPLS network performed better than IP network. That is because of the functionality of MPLS which utilizes all the paths to the destinations..IP network reached its steady state (in this case $0.5 \mathrm{Mbps}$ ) when the path (2-36-7-8) is saturated. Then, it started dropping packets; however, MPLS network reached its steady state (in this case $1 \mathrm{Mbps})$ when both paths (2-3-6-7-8) and (23-4-5-7-8) are saturated, then, it started dropping packets.

Referring to Table 3, it can be observed that MPLS technology reduced the packet loss rate from 81.32$73.45 \%$. In the beginning both IP network and MPLS network performed the same because both of them start building the information database, which we called routing tables in IP networks. In MPLS networks, Label Information Base (LIB) is used.

Referring to Table 4, it can be observed the total number of IPTV packets received at destination nodes in IP network is 461. In details, 265 packets received at Node 9, while 196 packets received at Node 10. In MPLS network the total number of IPTV packets received at destination nodes is 896. In details, 454 packets received at Node 9 , while 442 packets received at Node 10. That is because of the functionality of MPLS technology, which sends the packet from both paths (2-3-6-7-8) and (2-3-4-5-7-8); while, IP network sends packet only on one path (2-3-6-7-8).

VoIP scenario: Figure 12 shows that the performance of IP network reached the steady state (in this case $500 \mathrm{Kbps}$ ) when the shortest path was saturated. Thus, IP network at this point started dropping packets; however MPLS network did not drop packets since MPLS technology utilizes the routes available to the destinations. Thus, the packet loss rate is zero. Also, it is due to the nature of the traffic generator that has been used in this scenario which is Pareto on/off traffic (i.e., sometimes there is a burst flow and sometimes the system is idle).

Referring to Table 5, it can be observed that the total number of VoIP packets received at destination 
nodes in IP network is 2848 . In details, 1513 packets received at Node 9, while 1335 packets received at Node 10. In MPLS network, the total number of VoIP packets received at destination nodes is 3398 . In details, 1655 packets received at Node 9 , while 1743 packets received at Node 10. This can be observed from Table 6.

FTP scenario: Figure 13 shows that MPLS network performed better than IP network. That is because of the same reason which was mentioned earlier, which is the functionality of MPLS that utilizes all available paths to the destinations. The available paths in our case are path (2-3-6-7-8) and path (2-3-4-5-7-8).

The packet loss rate in this scenario is $0 \%$ for both MPLS network and IP network. That is because the main goal of this scenario is to show how MPLS technology speeds up network traffic flow based on the total number of packets sent as an indicator. It has been observed that the number of sent packets is 6488 in IP network; while in MPLS network the number of sent packets is 12259 .

The total number of FTP packets received at destination nodes in IP network is 534. In details, 267 packets received at Node 9, while 267 packets received at Node 10.

Referring to Table 7, we observed that in MPLS network, the total number of FTP packets received at destination nodes is 977 . In details, 463 packets received at Node 9, while 514 packets received at Node 10.

Related works: Differential Services (DiffServ) (Zarifzadeh et al., 2007) is a computer networking architecture that specifies a simple and scalable mechanism for classifying, managing network traffic and providing QoS guarantees on modern IP networks; however in the competition of DiffServ and MPLS, MPLS has been emerging as the protocol of the NGN for many reasons such as that MPLS is a multi protocol technology, where it can work over ATM, FR, etc. Also, MPLS is capable of providing controllable QoS features (Urra et al., 2006) by utilizing Classification, Queue and Scheduling (CQS) which enables high quality end-to-end service features that are necessary in applications such as VPN (Chung et al., 2001).

Performance analysis of the behavior of MPLS protocols has been done by Rahman et al. (2008), where a simulation environment is created for traditional IP and MPLS. Rahman et al. (2008) compared in their experiment between RSVP and CRLDP. They observed that RSVP has drawbacks in scalability compared to CR-LDP which performs better. It generally concluded that MPLS technology improves the packet transmissions in terms of delay and loss. However Rahman et al. (2008) project was on MPLS signaling protocol, there was no justification for the conclusion about the scalability of CRLDP compared to RSVP. Rahman et al. (2008) only ran simulations for comparing traditional IP network and MPLS network. On the other hand, a traffic analysis of MPLS and non MPLS network including MPLS signaling protocols has been done by Porwal et al. (2008), where the CR-LDP, RSVP and RSVP-TE MPLS signaling protocols have been compared based on how to setup LSP tunnels for TE with the help of the protocol messages. It has been concluded that RSVP has a drawback in its scalability when there are a large number of paths passing through a node due to the periodical refreshing of the state for each path. Also, a simulation environment is created for traditional IP and MPLS. It can be observed that MPLS signaling protocol that has been used was not declared. The authors have not justified their conclusion which was that when MPLS TE applied to the network, the performance of the network is significantly improved.

In (Lai et al., 2008), a method has been proposed, which attempts to establish all possible bypass tunnels based on the available bandwidth between two LSRs around the protected label switched router in MPLS. When a link or a LSR is broken, the LSR that detects the failure chooses a bypass tunnel to reroute traffic for each affected LSPs. The simulation results indicate that the proposed method has less packet losses in rerouting and can allow more affected LSPs to reroute traffic than RSVP.

In (Kocak et al., 2009), some fundamental aspects of MPLS over ATM method, IP over ATM method and multimedia application traffics with different QoS requirements have been presented. A simulation tool has been used to show that MPLS over ATM method provides a support for Average Bit Rate (ABR) QoS, Constant Bit Rate (CBR) QoS, Variable Bit Rate (VBR) QoS and a primitive UBR QoS for transferring Triple Play Services traffics. It has been concluded that MPLS over ATM method provides improved results for all of the multimedia traffics. Moreover, it overcomes the disadvantages of the IP over ATM method for producing erratic results for the data, voice and video application traffics. It can be observed that Kocak et al. (2009) focused on the ATM. Heterogeneous streams, which results due to issues such as disparate traffic characteristics of each stream, or competing customers' traffic, raise the issue of whether to multiplex some of these streams. In an MPLS network, such multiplexing 
can be considered by putting different streams into a tunnel identified by a single LSP, assuming that the different LSPs are assigned a reserved share of the resources. This point becomes even more important in the TE of a backbone network when a decision needs to be made on which streams have to be multiplexed, especially when there are constraints on tunneling, capacity and routing requirements for tunnels. This problem has been addressed in (Srivastava et al., 2009).

In (Klopfenstein, 2008), mathematical models were introduced and analyzed for addressing the problem of rerouting tunnels in an MPLS network in order to improve the resource utilization, where three levels of QoS have been considered, with different associated types of LSPs. A global rerouting framework is proposed, which enabled independently the consideration each type of LSP.

\section{CONCLUSION}

Traditional IP networks have many limitations such as routing tables which can be complex and time consuming. Thus, it offers little predictability of service, which is unacceptable for triple play services. MPLS technology has been proposed to overcome these limitations, to speed up the traffic flow and also can provide a $\mathrm{QoS}$ for real time applications by using labels. In this research, the performance of MPLS for improving triple play services has been evaluated and compared to that provided by traditional IP networks using VoIP, IPTV and FTP applications using NS-2 simulation. The average throughput of the network, number of packets received at destination nodes and packet loss rates have been considered as performance metrics. After running the simulations, it has been observed that MPLS technology performed better than IP networks in all scenarios. Thus, it improves the performance of the network in heavy traffic environments, which allows ISPs to provide better triple play services.

Validating the findings of this work using test bed is one of our suggestions for future work. It will be interesting to see how these networks work in a real environment and how these networks react when security threats are applied in a real environment. From the literature review, we observed that most researches have been done in the area of label distribution protocols. Thus, we suggest that the infrastructure of labeling mechanism in MPLS technology which might consume the bandwidth in signaling should be studied in more details. The importance of MPLS technology to the NGN is also an area which can be considered as a future research.

\section{REFERENCES}

Banerjee, A., J. Drake, J. Lang, B. Turner and D. Awduche et al., 2001. Generalized multiprotocol label switching: An overview of signaling enhancements and recovery techniques. IEEE Commun. Mag., 39: 144-151. DOI: $10.1109 / 35.933450$

Buckwalter, J.T., 2000. Frame Relay: Technology and Practice. Addison-Wesley Professional, ISBN: 0201485249 , pp: 338.

Chow, H.K. and A. Leon-Garcia, 1999. Integrated services internet with RSVP over ATM short cuts: Implementation and performance evaluation. Comput. Commun., 22: 792-802. DOI: 10.1016/S0140-3664(99)00047-X

Chung, J.M., S.C. Kim, E. Marroun and H. Sandhu, 2001. VoIP over MPLS Networking Requirements. In: Networking-ICN, Lorenz, P. (Ed.). SpringerVerlag, ISBN: 9783540423034, pp: 735-744.

Comer, D.E., 2000. Internetworking with TCP/IP: Principles, Protocols and Architectures. 4th Edn., Prentice Hall, ISBN: 10: 0130183806, pp: 750.

Cuthbert, L.G. and J.C. Sapanel, 1993. ATM: The Broadband Telecommunications Solution. Institution of Engineering and Technology, ISBN: 0852968159, pp: 161.

Doyle, J. and J.D. Carroll, 2001. Routing TCP/IP, Volume II. Cisco Press, ISBN: 1578700892, pp: 976.

El Hachimi, M., A. Abouaissa, P. Lorenz and M.O. Lee, 2004. Scalable multicast provisioning in diffServ with MPLS labeling. Telecommun. Syst., 27: 253-272. DOI: 10.1023/B:TELS.0000041011.21556.da

Feher, G., K. Nemeth and I. Cselenyi, 2002. Performance evaluation framework for IP resource reservation signaling. Perform. Evaluat., 48: 131-156. DOI: $10.1016 / S 0166-5316(02) 00034-2$

Goralski, W., 2009. The Illustrated Network: How TCP/IP Works in a Modern Network. Morgan Kaufmann, Boston, ISBN: 9780123745415, pp: 797.

Klopfenstein, O., 2008. Rerouting tunnels for MPLS network resource optimization. Eur. J. Operat. Res., $\quad 188$ : 293-312. DOI: 10.1016/j.ejor.2007.04.016

Kocak, C., I. Erturk and H. Ekiz, 2009. MPLS over ATM and IP over ATM methods for multimedia applications. Comput. Stand. Interfaces, 31: 153160. DOI: $10.1016 / j . c s i .2007 .11 .003$

Lai, W.K., Z.C. Zheng and C.D. Tsai, 2008. Fast reroute with pre-established bypass tunnel in MPLS. Comput. Commun., 31: 1660-1671. DOI: 10.1016/j.comcom.2007.11.008 
Lee, Y.W., S. Kim, J. Park and S.H. Kim, 2007. A lightweight implementation of RSVP-TE protocol for MPLS-TE signaling. Comput. Commun., 30: 1199-1204. DOI: 10.1016/j.comcom.2006.12.003

Loshin, P., 2003. The Evolution of File Transfer Protocol: TCP/IP Clearly Explained. 4th Edn., Morgan Kaufmann, San Francisco, ISBN: 9781558607828, pp: 709.

Issariyakul, T. and E. Hossain, 2008. Introduction to Network Simulator NS2. Springer US, ISBN: 1441944125, pp: 435.

Karapantazis, S. and F.N. Pavlidou, 2009. VoIP: A comprehensive survey on a promising technology. Comput. Networks, 53: 2050-2090. DOI: 10.1016/j.comnet.2009.03.010

Martin, R., 2008. Multiprotocol Label Switching. In: The Cable and Telecommunications Professionals' Reference, Hill, G. (Ed)., 3rd Edn., Focal Press, Boston, ISBN: 9780240807485, pp: 377-402.

Maufer, T.A., 1999. IP Fundamentals: What Everyone Needs to Know About Addressing and Routing. Prentice Hall, ISBN: 0139754830, pp: 450.

Mortada, I. and W. Probst, 2001. Internet telephony signaling. Telemat. Inform., 18: 159-194. DOI: 10.1016/S0736-5853(00)00027-7

Porwal, M.K., A. Yadav and S.V. Charhate, 2008. Traffic analysis of MPLS and non MPLS network including MPLS signaling protocols and traffic distribution in OSPF and MPLS. Proceedings of the 1st International Conference on Emerging Trends in Engineering and Technology, July 16-18, IEEE Xplore Press, Nagpur, Maharashtra, pp: 187-192. DOI: 10.1109/ICETET.2008.58

Rahman, M.A., A.H. Kabir, K.A.M. Lutfullah, M.Z. Hassan and M.R. Amin, 2008. Performance analysis and the study of the behavior of MPLS protocols. Proceeding of the International Conference on Computer and Communication Engineering, May 13-15, IEEE Xplore Press, Kuala Lumpur, pp: 226-229. DOI: 10.1109/ICCCE.2008.4580601
Simpson, W., 2008. Video Over IP: IPTV, Internet Video, H.264, P2P, Web TV, and Streaming: A Complete Guide to Understanding the Technology. 2nd Edn., Elservier, ISBN: 0240810848, pp: 478.

Sridharan, A., R. Guerin C. Diot, 2005. Achieving nearoptimal traffic engineering solutions for current OSPF/IS-IS networks. IEEE/ACM Trans. Network., 13: 234-247. DOI: 10.1109/TNET.2005.845549

Srivastava, S., A. van de Liefvoort and D. Medhi, 2009. Traffic engineering of MPLS backbone networks in the presence of heterogeneous streams. Comput. Networks: Int. J. Comput. Telecommun. Network., 53: 2688-2702. DOI: 10.1016/j.comnet.2009.06.003

Szviatovszki, B., A. Szentesi and A. Juttner, 2002. Minimizing re-routing in MPLS networks with preemption-aware constraint-based routing. Comput. Commun., 25: 1076-1084. DOI: 10.1016/S0140-3664(02)00025-7

Tiwari, A. and A. Sahoo, 2007. Providing QoS in OSPF based best effort network using load sensitive routing. Simulat. Model. Pract. Theor., 15: 426-448. DOI:10.1016/j.simpat.2006.11.008

Urra, A., E. Calle and J.L. Marzo, 2006. Reliable services with fast protection in IP/MPLS over optical networks. J. Opt. Network., 5: 870-880. DOI: 10.1109/TNET.2007.900411

Wang, D. and G. Li, 2008. Efficient distributed bandwidth management for MPLS fast reroute. IEEE/ACM Trans. Network., pp: 486-495. DOI: 10.1109/TNET.2007.900411

Zarifzadeh, S., N. Yazdani and H. Khanmirza, 2007. A routing framework for load balancing of bandwidth sensitive traffic in differentiated service networks. Comput. Networks, 51: 1183-1204. DOI: 10.1016/j.comnet.2006.07.010 